\title{
THE COUNTABLE NEIGHBOURHOOD PROPERTY AND TENSOR PRODUCTS
}

\author{
by JOSÉ BONET
}

(Received 21st June 1984)

This article is intended to enlarge the study of spaces satisfying the countable neighbourhood property and to clarify the incidence of this property in the stability of some locally convex properties of tensor products.

We shall use the standard notations of locally convex spaces as in [17] and [18]. The word space will always mean separated locally convex space. If $(E, t)$ is a space, the set of all continuous seminorms on it will be denoted by $\operatorname{cs}(E)$. The linear hull and the absolutely convex hull of a subset $C$ of a space will be written lin $(C)$ and $\Gamma(C)$ respectively. If $(E, t)$ is a space and $F$ is a subspace of $E$, by $(F, t)$ we mean $F$ endowed with the topology induced by $t$, which we denote $t \mid F$ when we refer to it. If $t$ and $t^{\prime}$ are two topologies on $E$ we write $t<t^{\prime}$ if $t$ is coarser than $t^{\prime}$. If $E$ and $F$ are spaces and $\mathscr{A}$ is a saturated family of bounded subsets of $E$ covering it, we denote by $L_{\mathscr{A}}(E, F)$ the space of linear continuous operators from $E$ into $F$ endowed with the topology of uniform convergence on the elements of $\mathscr{A}$. We will denote by $E \varepsilon F$ the $\varepsilon$-product of Schwartz of the spaces $E$ and $F$ as in [18, vol. II, p. 242].

A space $E$ is said to satisfy the countable neighbourhood property (c.n.p.) if for every sequence $\left(U_{n}\right)$ of 0 -neighbourhoods in $E$ there are $c_{n}>0$ such that $U=\cap_{n=1}^{\infty} c_{n} U_{n}$ is a 0 neighbourhood in $E$, or equivalently if for every sequence $\left(p_{n}\right)$ in $\operatorname{cs}(E)$ there are $c_{n}>0$ and $p \in \operatorname{cs}(E)$ such that $p_{n} \leqq c_{n} p, n=1,2, \ldots$ (see $[11,4.8$.]). Floret proved in [11] that the class of spaces satisfying the c.n.p. is stable under forming subspaces, quotients, completions and countable inductive limits. A large and useful class of spaces enjoying this property is provided by the ( $g D F)$-spaces. An exhaustive study of $(g D F)$ and $(d f)$ spaces can be found in [17, Ch. 12], and we refer the reader to it.

Spaces satisfying the c.n.p. have appeared widely in applications. They appear with the name of class $(Q)$ in the book of De Wilde [9] in a study of the stability of spaces with C-web and Suslin spaces. They have been used in infinite holomorphy (see [6] and [22] for instance). Their incidence in the commutability of inductive limits and tensor products occurs in [11], [14] and [15]. Spaces satisfying the c.n.p. are utilized in the study of compactness and weak compactness of sets of operators in [24] and [25]. In [10] S. Dierolf extensively studies the c.n.p. in the context of spaces of continuous linear mappings. Colombeau assumes the c.n.p. and other condition in a real space to obtain extensions of the classical Borel Theorem on $C^{\infty}$ functions with a given sequence of derivatives at a certain point in [5]. Recently in [2] we were able to extend some projective descriptions of weighted inductive limits given in [1] to spaces of continuous functions with values in spaces satisfying the c.n.p. In view of all this it is our impression 
that spaces satisfying the c.n.p. deserve some attention. In Section 1 we recall some hereditary properties, and include some counter-examples clarifying the relation of the c.n.p. and $(g D F)$-spaces, answering questions of Hollstein and Ruess. In Section 2 we give a characterization in terms of tensor products and applications of it.

\section{Properties and examples}

1.1. The following properties can be easily verified: (i) A metrizable space satisfies the c.n.p. if and only if it is normable.

(ii) Let $E$ and $F$ be spaces and $\mathscr{A}$ a saturated family of bounded subsets of $E$ covering it. The space $L_{\mathscr{A}}(E, F)$ satisfies the c.n.p. if and only if $F$ and $E^{\prime}$ endowed with the topology of the uniform convergence on the elements of $\mathscr{A}$ satisfy the c.n.p.

(iii) If $E$ and $F$ are spaces satisfying the c.n.p., then $E \otimes_{\pi} F, E \otimes_{\varepsilon} F, E \otimes_{\pi} F$ and $E \varepsilon F$ satisfy the c.n.p.

(iv) Let $E$ be a space, then $\left(E, \sigma\left(E, E^{\prime}\right)\right)$ satisfies the c.n.p. if and only if $E$ is finite dimensional.

(v) If $X$ is a Hausdorff completely regular space, then the space of continuous functions on $X$ endowed with the compact open topology is a (DF)-space if and only if it satisfies the c.n.p.

1.2. By a three-space-problem we understand the following situation: Let $F$ be a closed subspace of a space $(E, t)$. Suppose that $(F, t)$ and $(E / F, \hat{t})$ satisfy a certain property $(P)$, does $(E, t)$ satisfy property $(P)$ ?. The example 3.5 in [23] shows that the three-space-problem is in general not true for $(g D F)$-spaces. In contrast to this we have that it is true for $(P)=$ c.n.p. A proof of this result can be seen in $[10,2.4$.].

1.3. The class of spaces satisfying the c.n.p. which are hereditary Lindelöf seems to be important in infinite holomorphy, since every open subset of a space with these properties is "uniformly open", i.e. there is $p \in c s(E)$ such that it is open in the seminormed space $(E, p)$ (see [6]). Since every open subset of a Suslin space is Lindelöff, the class $(Q S)$ of all,Suslin spaces satisfying the c.n.p. is a rich subclass of the original one with "good" hereditary properties. More precisely, recalling the properties of Suslin spaces included in [28, Ch. I \$4.4] and [9, Ch. VII], we have: (i) Strong duals of Fréchet Montel spaces and countable inductive limits of separable Banach spaces belong to $(Q S)$. (ii) The dual of every metrizable separable space endowed with the topology of precompact convergence belongs to $(Q S)$. (iii) The class $(Q S)$ is stable under forming separated quotients and countable inductive limits. (iv) The projective and injective tensor product of spaces belonging to $(Q S)$ belong to $(Q S)$. This is a consequence of 1.1 (iii) and [27, Theorem 3 and Corollary 1.3]. (v) if $E$ is a separable metrizable space and $F$ is a separable Banach space, then $L_{c}(E, F)$ belong to $(Q S)$, where $c$ is the class of precompact subsets of $E$. (vi) if $E$ is a separable Fréchet space and $F$ is a sequentially complete space in $(Q S)$, then $L_{c}(E, F)$ belongs to $(Q S)$. (vii) If $E$ is a separable Fréchet space and $F$ is a complete space in $(Q S)$, then $E_{c}^{\prime} \hat{\otimes}_{\varepsilon} F$ belongs to $(Q S)$.

1.4. A space satisfying the c.n.p. without a fundamental system of bounded sets.

In [11] Floret gave an example of this type which was not correct. We modify his 
idea to obtain a new example: Let $I$ be a non countable set of indices. We set

$$
\begin{array}{cl}
K^{(I)}=\left\{x \in K^{I} \mid\{i \in I \mid x(i) \neq 0\}\right. & \text { is finite }\} \\
K^{[I]}=\left\{u \in K^{I} \mid\{i \in I \mid u(i) \neq 0\}\right. & \text { is countable }\} .
\end{array}
$$

For every $u \in K^{[I]}$ we define

$$
p_{u}(x)=\sum(|x(i) u(i)|: i \in I), \quad \text { for each } x \in K^{(I)}
$$

We consider the space $K^{(I)}$ endowed with the locally convex topology defined by the system of seminorms $\left\{p_{u} \mid u \in K^{[I]}\right\}$.

Let $B$ be a bounded subset of $K^{(I)}$. Then there is a finite subset $J$ of $I$ such that $x(i)=0$ for every $x \in B$ and $i \in I \backslash J$. If it is not true we can determine a sequence $\left(x_{n}\right)$ in $B$ and a sequence $\left(i_{n}\right)$ in $I$ such that $x_{n}\left(i_{n}\right) \neq 0, n=1,2, \ldots$ For every positive integer $n$, let $y_{n}$ be the element of $K^{(I)}$ such that $y_{n}\left(i_{n}\right)=x_{n}\left(i_{n}\right)$ and $y_{n}(i)=0$ if $i \neq i_{n}$. The set $D=$ $\left\{y_{n} \mid n=1,2, \ldots\right\}$ is bounded in $K^{(I)}$ since $B$ is bounded. Now taking $v \in K^{[I]}$ defined by $v(i)=n\left|x_{n}\left(i_{n}\right)\right|^{-1}$ if $i=i_{n}, n=1,2, \ldots$, and $v(i)=0 \quad i \in I, i \neq i_{n}, n=1,2, \ldots$, we have that $p_{v}\left(y_{n}\right)=n, n=1,2, \ldots$, which is a contradiction. Thus $K^{(I)}$ can not be covered by a sequence of bounded subsets.

We shall see that $K^{(I)}$ satisfies the c.n.p. We take a sequence $\left(u_{k}\right)$ in $K^{[I]}$ and we set $I_{k}=\left\{i \in I \mid u_{k}(i) \neq 0\right\}$ and $J=\cup_{k=1}^{\infty} I_{k}$, which is a countable subset of $I$. We put $v_{k}$ to denote the element of $K^{J}$ restriction of $u_{k}$ to $J$. The topology of $K^{(I)}$ induces on $K^{(J)}$ the finest locally convex topology, which coincides with the normal topology with respect to its $\alpha$ dual $K^{J}$ (see [18, Vol. I, p. 406]), and satisfies the c.n.p. since it is a $(D F)$-space. Thus we can determine $w \in K^{J}$ and $c_{k}>0$ such that

$$
\sum\left(\left|x(i) v_{k}(i)\right|: i \in J\right) \leqq c_{k} \sum(|x(i) w(i)|: i \in J)
$$

for every $x \in K^{(J)}$ and $k=1,2, \ldots$

If we take now $v \in K^{[J]}$ defined by $v(i)=w(i)$ if $i \in J$ and $v(i)=0$ if $i \in I \backslash J$, we have that

$$
p_{u_{k}}(x) \leqq c_{k} p_{v}(x) \text { for every } x \in K^{(I)} \text { and } k=1, \overline{2}, \ldots
$$

1.5 A closed subspace of a $(g D F)$-space which is not a $(g D F)$-space but is a $(d f)$-space.

Semi-Montel $(g D F)$-spaces are called $(D C F)$-spaces by Hollstein in [14]. It is proved therc that every closed subspace of a $(D C F)$-space is also a $(D C F)$-space. The situation for $(g D F)$-spaces is different.

We set $G=\left(\ell^{p}\right)^{(N)}$, the countable direct sum of copies of the Banach space $\ell^{p}$, with $1<p<\infty$, and $G_{n}=\oplus_{j=1}^{n} \ell^{p}$. There is a classical example of Grothendieck, [12], of a closed subspace $F$ of $G$ such that the topology $t$ induced by $G$ is strictly coarser than the topology $t^{\prime}$ of the inductive limit ind $F \cap G_{n}$. In this case it is easy to see that the topologies $t$ and $t^{\prime}$ have the same bounded subsets and coincide on them. Hence $(F, t)$ is not a $(g D F)$-space. On the other hand, since $G$ is a reflexive $(L B)$-space we have that $(F, t)$ is a $(d f)$-space (see [16, p. 262]) and satisfies the c.n.p. as a subspace of $G$.

A similar situation has been considered in [19]. 
1.6. A space satisfying the c.n.p. with a fundamental system of bounded sets whose strong dual is not locally complete.

Grothendieck in [12] gives an example of a closed subspace $F$ of $E=\left(\ell^{\infty}\right)^{(N)}$ such that if $t$ is the topology induced by $E$ on $F$ and $t^{\prime}$ the topology of the inductive limit of the sequence $F_{n}=F \cap\left(\oplus_{j=1}^{n} \ell^{\infty}\right), n=1,2, \ldots$, there is a linear form $g$ on $F$ which is $t^{\prime}$ continuous but not $t$-continuous (see also [28, Ch. II $\S 5,7]$ ). Clearly $(F, t)$ is a space satisfying the c.n.p. with a fundamental system of bounded sets. Moreover $t\left|F_{n}=t^{\prime}\right| F_{n}$, $n=1,2, \ldots$. Thus for every positive integer $n$ the restriction of $g$ to $\left(F_{n}, t \mid F_{n}\right)$ is linear and continuous, therefore there is a continuous linear form $f_{n}$ on $(F, t)$ whose restriction to $F_{n}$ coincides with $g$. Since every bounded subset of $(F, t)$ is localized in some $F_{n}$, we have that the sequence $n\left(f_{n}-g\right)$ converges to zero uniformly on the bounded subsets of $(F, t)$. Then the strong dual of $(F, t)$ is not locally complete.

In this case the strong dual of $(F, t)$ is a non-complete metrizable space, and therefore $(F, t)$ is not a $(d f)$-space.

We observe that this example shows that the countable neighbourhood property is not a "quasi-barrelled" type condition.

1.7. An $\ell^{\infty}$-barrelled space with a fundamental system of bounded sets, hence a (df)space, which doed not satisfy the c.n.p.

Ruess in [25, p. 436] says that it is not known if spaces with these properties exist.

We consider an index set $I=\cup_{n=1}^{\infty} I_{n}$, with $I_{n} \subset I_{n+1}$ and $I_{n+1} \backslash I_{n}$ not countable. We take $E=\ell^{2}(I)$ and consider the following seminorms: for a separable weakly bounded subset $A$ of $E^{\prime}=\ell^{2}(I)$, we set

$$
p_{A}(x)=\sup \{|\langle x, u\rangle|: u \in A\}, x \in E,
$$

and for every positive integer $n$ we set

$$
p_{n}(x)=\left(\sum\left(|x(i)|^{2}: i \in I_{n}\right)\right)^{1 / 2}, x \in E .
$$

We denote by $t$ the locally convex topology in $E$ generated by the family of seminorms

$$
\left\{p_{n} \mid n=1,2, \ldots\right\} \cup\left\{p_{A} \mid A \subset E^{\prime} \quad \text { weakly bounded and separable }\right\} .
$$

Clearly $(E, t)$ is $\ell^{\infty}$-barrelled and has a fundamental system of bounded sets.

Suppose that $(E, t)$ satisfies the c.n.p., there are $c_{n}>0, n=1,2, \ldots$, and $p \in c s(E)$ such that

$$
p_{n}(x) \leqq c_{n} p(x) \text { for every } x \in E
$$

Therefore $p$ is a continuous norm on $E$, but this is not possible. Indeed, since $p$ is a continuous norm there exist a positive integer $k$, a positive constant $C$ and a separable weakly bounded subset $A$ of $E^{\prime}$ such that $p \leqq C\left(p_{k}+p_{A}\right)$. Since $A$ is separable and every element of $\ell^{2}(I)$ has countably many coordinates distinct from zero, we can determine a countable subset $J$ of $I$ such that $u(i)=0$ for every $i \in I \backslash J$ and $u \in A$. By the very construction there exists $i_{0} \in I \backslash\left(I_{k} \cup J\right)$. Taking the element $y$ in $E$ with $y\left(i_{0}\right)=1$ and $y(i)=0$ 
if $i=i_{0}$, we obtain that

$$
0<p(y) \leqq C\left\{\sup \{|\langle y, u\rangle|: u \in A\}+p_{k}(y)\right\}=0
$$

which is a contradiction.

This example appears in [3] with a different purpose and it is a modification of another one given in [19].

\section{Incidence in tensor products}

In [11] Floret proves that for every tensor norm topology $\alpha$, if $E$ is a space satisfying the c.n.p. and $\left(F_{n}\right)$ is a sequence of spaces, then the algebraic equality $E \otimes_{\alpha}\left(\oplus_{n=1}^{\infty} F_{n}\right)=$ $\oplus_{n=1}^{\infty}\left(E \oplus_{\alpha} F_{n}\right)$ holds also topologically. This is a result of Grothendieck for $\alpha=\pi, \varepsilon$ and $E$ a $(D F)$-space. In [18] Köthe, with the same conditions, proves that $\left(\oplus_{n=1}^{\infty} F_{n}\right) \varepsilon E$ is isomorphic to $\bigoplus_{n=1}^{\infty}\left(F_{n} \varepsilon E\right)$. We shall show that the class of spaces satisfying the c.n.p. is in some sense the best possible in such theorems.

Theorem 2.1. A space E satisfies the c.n.p. if and only if $E \otimes_{\pi} K^{(N)}=E^{(N)}$ holds topologically.

Proof. The necessary condition is the result of Grothendieck. Conversely we consider $E \otimes_{\pi} K^{(N)}$ and $E^{(N)}$ algebraically equal by means of the linearization of the bilinear mapping $B$ from $E \times K^{(N)}$ into $E^{(N)}$ defined by $B\left(x,\left(a_{n}\right)\right)=\left(a_{n} x\right)$, for every $x \in E$ and $\left(a_{n}\right) \in K^{(N)}$. The equality holds topologically if and only if $B$ is continuous. We take now a sequence $\left(U_{n}\right)$ of absolutely convex 0 -neighbourhoods in $E$. By $\left[18, \S 18,5\right.$. (8)] $\oplus_{n=1}^{\infty} U_{n}$ is a 0 -neighbourhood in $E^{(N)}$. Since $B$ is continuous there are $c_{n}>0, n=1,2, \ldots$ and an absolutely convex 0 -neighbourhood $U$ such that, if $V\left(c_{n}\right)=\left\{a \in K|| a \mid \leqq c_{n}\right\}, n=1,2, \ldots$, we have that

$$
B\left(U \times\left(\bigoplus_{n=1}^{\infty} V\left(c_{n}\right)\right)\right) \subset \bigoplus_{n=1}^{\infty} U_{n}
$$

from where it follows that

$$
U \subset \bigcap_{n=1}^{\infty} c_{n}^{-1} U_{n}
$$

and hence $E$ satisfies the c.n.p. This completes the proof.

By a well-known result of Hollstein $K^{N} \otimes_{\pi} K^{(N)}$ is not barrelled (see f.i. [17, p. 333]). In [4] we prove that for a metrizable space $E, E \otimes_{\pi} K^{(N)}$ is barrelled if and only if $E$ is normable. In view of 1.1 (i) our next corollary improves this result.

Corollary 2.2. Let $E$ be a space. $E \otimes_{\pi} K^{(N)}$ is barrelled if and only if $E$ is barrelled and satisfies the c.n.p.

Proof. If $E$ is barrelled and satisfies the c.n.p., then $E \otimes_{\pi} K^{(N)}=E^{(N)}$ is also barrelled. Conversely the barrelled space $E \otimes_{\pi} K^{(N)}$ is the increasing union of the spaces $G_{n}=$ 
$E \otimes \oplus_{j=1}^{n} K, n=1,2, \ldots$ The space $G_{n}$ endowed with the induced topology coincides with $\oplus_{j=1}^{n} E$ algebraically and topologically. By [28, Ch. I $\left.\S 3,1 .(9)\right]$, the space $E \bigotimes_{\pi} K^{(N)}$ is the inductive limit of the sequence $\left(G_{n}\right)$, and Theorem 2.1 can be applied. This completes the proof.

Proposition 2.3. Let $F$ be a barrelled (DF)-space with no total bounded subset, and $E$ a space. Then $E \otimes_{\pi} F$ is barrelled if and only if $E$ is barrelled and satisfies the c.n.p.

Proof. If $F$ is barrelled $(D F)$-space with no total bounded subset, then $F$ contains a complemented subspace isomorphic to $K^{(N)}$. Therefore if $E \otimes_{\pi} F$ is barrelled, then $E \otimes_{\pi} K^{(N)}$ is barrelled and we can apply Corollary 2.2 . Conversely let $\left(B_{n}\right)$ be a fundamental sequence of bounded subsets of $F$ and $T$ a barrel in $E \otimes_{\pi} F$. We set

$$
V_{n}=\left\{x \in E \mid x \otimes y \in T \text { for every } y \in B_{n}\right\} .
$$

The set $V_{n}$ is absolutely convex and closed in $E$. Moreover it is absorbent. Indeed, take $z \in E$ and set

$$
G(z)=\{y \in F \mid z \otimes y \in T\}
$$

which is an absorbent closed absolutely convex subset of $F$, and hence a 0 neighbourhood in $F$. Thus there is a $>0$ such that $B_{n} \subset a G(z)$, and consequently $z \in a V_{n}$. Since $E$ is barrelled, $V_{n}$ is a 0 -neighbourhood in $E, n=1,2, \ldots$ We apply the c.n.p. to obtain an absolutely convex 0-neighbourhood $V$ in $E$ and $c_{n}>0, n=1,2, \ldots$, such that $V \subset \cap_{n=1}^{\infty} c_{n} V_{n}$. Now the set $U=\overline{\Gamma\left(\cup_{n=1}^{\infty} c_{n}^{-1} B_{n}\right)}$ is a 0 -neighbourhood in $F$ and $\Gamma(V \otimes U) \subset T$. This completes the proof.

The following result clarifies our former Proposition. It is a special case of $[7$, Theorem 6].

Proposition 2.4. Let $E$ be a space satisfying the c.n.p. and $F$ a $(D F)$-space. If $E$ and $F$ are barrelled (resp. bornological, quasi-barrelled), then $E \otimes_{\pi} F$ is barrelled (resp. bornological, quasi-barrelled).

Now we shall give an extension of Hollstein's abstract version of the theorem of Mujica [21] on the commutability of inductive limits and injective tensor products. We refer the reader to [15] for $\varepsilon$-spaces and to [16] for inductive limits with a local partition of unity.

Proposition 2.5. Let $E$ be a space satisfying the c.n.p. and the Schwartz approximation property. Let $F=\operatorname{ind} F_{n}$ be a separated inductive limit. If

(i) $E$ is an $\varepsilon$-space, or

(ii) $F$ has a local partition of unity,

then ind $E \varepsilon F_{n}$ is isomorphic to a dense topological subspace of $E \varepsilon$ ind $F_{n}$. 
Proof. Let $j_{n}: F_{n} \rightarrow F$ and $j_{n, n+1}: F_{n} \rightarrow F_{n+1}$ be the canonical injections. The mappings $\mathrm{id}_{E} \varepsilon j_{n}: E \varepsilon F_{n} \rightarrow E \varepsilon F$ and $\mathrm{id}_{E} \varepsilon j_{n, n+1}: E \varepsilon F_{n} \rightarrow E \varepsilon F_{n+1}$ are injective, linear, continuous and satisfy $\left(\mathrm{id}_{E} \varepsilon j_{n+1}\right) \circ\left(\mathrm{id}_{E} \varepsilon j_{n, n+1}\right)=\mathrm{id}_{E} \varepsilon j_{n}$. By the universal property of inductive limits there is a continuous linear injective mapping from ind $E \varepsilon F_{n}$ into $E \varepsilon F$. We prove that this mapping is also open. Since $E$ satisfies the Schwartz approximation property, $E \otimes F$ is dense in $E \varepsilon F$ and $E \otimes F_{n}$ is dense in $E \varepsilon F_{n}, n=1,2, \ldots$, from where it follows that $\cup_{n=1}^{\infty} E \otimes F_{n}$, which can be identified $E \otimes F$, is a dense subspace of ind $E \varepsilon F_{n}$. We set $H=\cup_{n=1}^{\infty} E \varepsilon F_{n}$ and $L=E \otimes F$ considered as subspaces of $E \varepsilon F$. We denote by $t$ the topology of $E \varepsilon F$ and by $t^{\prime}$ the topology of ind $E \varepsilon F_{n}$ in $H$. Clearly $t^{\prime}$ is finer than the topology $t \mid H$, and $(L, t \mid L)$ coincides with $E \otimes_{\varepsilon} F$. Since the injections

$$
E \otimes_{\varepsilon} F_{n} \rightarrow E \varepsilon F_{n} \rightarrow\left(H, t^{\prime}\right), n=1,2, \ldots,
$$

are continuous, if we denote by $t^{\prime \prime}$ the topology on $L$ such that $\left(L_{1} t^{\prime \prime}\right)=$ ind $E \otimes_{\varepsilon} F_{n}$, we have that $t\left|L \prec t^{\prime}\right| L \prec t^{\prime \prime}$. Under condition (i) or (ii) $t \mid L$ coincides with $t^{\prime \prime}$, by [15, Proposition 4.3.] and [16, Proposition 3.2.] respectively. Since $t^{\prime}$ is finer than $t \mid H$ on $H$ and $L$ is a $t^{\prime}$-dense subspace of $H$ we can apply [1, Lemma 1.2] to obtain that $t^{\prime}=t \mid H$, and thus ind $E \varepsilon F_{n}$ is a topological subspace of $E \varepsilon F$. This completes the proof.

The Banach $\varepsilon$-spaces are the $\mathscr{L}^{\infty}$-spaces, [15], therefore we have

Corollary 2.6. If $E$ is an $\mathscr{L}^{\circ}$-space and $F=$ ind $F_{n}$ is a separated inductive limit, then ind $E \varepsilon F_{n}$ is a dense topological subspace of $E \varepsilon F$.

Corollary 2.7. If $E$ is an $\mathscr{L}^{\infty}$-space and $F$ is an $(L F)$-space, then $E \varepsilon F$ is barrelled.

Proof. By Corollary 2.6 EEF contains a dense subspace which is an $(L F)$-space and therefore barrelled. This completes the proof.

Now we consider the barrelledness or quasi-barrelledness of the $\varepsilon$-product of an $\mathscr{L}^{\infty}$ space and a barrelled or quasi-barrelled space respectively. In [7] Defant and Govaerts prove that if $E$ is an $\mathscr{L}^{\infty}$-space then $E \otimes_{e} F$ is quasi-barrelled if and only if $F$ is quasibarrelled and its strong dual $F_{b}^{\prime}$ has the property (B) of Pietsch. Modifying the proof of [7, Proposition 4] one can show that if $E$ is a space with the bounded approximation property, then $E \otimes_{\varepsilon} F$ is a large subspace of $E \varepsilon F$, i.e., every bounded subset of $E \varepsilon F$ is included in the closure of a bounded subset of $E \otimes_{\varepsilon} F$. Since every $\mathscr{L}^{\infty}$-space satisfies the bounded approximation property we obtain.

Proposition 2.8. If $E$ is an $\mathscr{L}^{\infty}$-space, then $E \varepsilon F$ is quasi-barrelled if and only if $F$ is quasi-barrelled and $F_{b}^{\prime}$ has the property $(B)$ of Pietsch.

Mendoza proves in [20] that $c_{0}(F)$ is barrelled if and only if $c_{0}(F)$ is quasi-barrelled and $F$ is barrelled. Defant and Govaerts ask in [8] if a similar result is true for $E \varepsilon F, E$ being an $\mathscr{L}^{\infty}$-space. This is not the case. By [26] every infinite dimensional separable Banach space contains a dense hyperplane $F$ whose absolutely convex compact subsets are finite dimensional. In this case $F$ is barrelled and $E \varepsilon F$ coincides with $E \otimes_{\varepsilon} F$ which is 
quasi-barrelled but not barrelled if $E$ is an infinite dimensional $\mathscr{L}^{\infty}$-space, as can be seen applying $[17,21.3 .3]$ (a situation also considered in $[10,4.9]$ ). A general result can be obtained with a completeness assumption on $F$.

Proposition 2.9. If $E$ is an $\mathscr{L}^{\infty}$-space and $F$ is a locally complete space, then $E \varepsilon F$ is barrelled if and only if $F$ is barrelled and $F_{b}^{\prime}$ has the property $(B)$ of Pietsch.

Proof. If $F$ is locally complete, then $E \varepsilon F$ is locally complete by [8, Proposition 1.3]. Since every locally complete quasi-barrelled space is barrelled, it is enough to apply Proposition 2.8. This completes the proof.

Remark 2.10. (a) Proposition 2.9 does not cover completely the barrelledness of the $\varepsilon$-pdoduct of an $\mathscr{L}^{\infty}$-space and a barrelled space. If $F$ is a non regular $(L F)$-space, then $E \varepsilon F$ is barrelled by Proposition 2.7 , but $F$ is not locally complete.

(b) The spaces $c_{0} \varepsilon F$ and $c_{0}(F)$ are different in general. More precisely, the mapping $A: c_{0} \varepsilon F \rightarrow c_{0}(F)$ defined by $A(u)=\left(u\left(e_{n}^{\prime}\right)\right)$, for every $u \in c_{0} \varepsilon F$, where $e_{n}^{\prime}, n=1,2, \ldots$, are the canonical unit vectors of $\ell^{1}=c_{0}^{\prime}$, is a topological isomorphism into a sequentially dense subspace of $c_{0}(F)$, and $A$ is onto if and only if $F$ is locally complete.

\section{REFERENCES}

1. K. Bierstedt, R. Meise, and W. H. Summers, A projective description of weighted inductive limits, Trans. Amer. Math. Soc. 272 (1982), 107-160.

2. J. BONET, A projective description of weighted inductive limits of vector valued continuous functions, Collect. Math. 34 (1983), 115-124.

3. J. Bonet and P. Pérez Carreras, Remarks on the stability of barrelled type topologies, Bull. Soc. Roy. Sci. Liège 52 (1983), 313-318.

4. J. Bonet and P. Perez Carreras, Some results on barelledness in projective tensor products, Math. Z. 185 (1984), 333-338.

5. J. F. Colombeau, Infinite dimensional $\mathrm{C}^{\infty}$ mapping with a given sequence of derivatives at a given point, J. Math. Anal. Appl. 71 (1979), 95-104.

6. J. F. Colombeau and J. Mujica, Existence of holomorphic mappings with prescribed asymptotic expansion at a given set of points in infinite dimensions, Nonlinear Analysis, Theory, Methods and Applications 5 (1981), 149-156.

7. A. Defant and W. Govaerts, Tensor products and spaces of vector valued continuous functions (preprint, 1983).

8. A. Defant and W. Govaerts, Bornological and ultrabornological spaces of type $C(X, F)$ and EEF, Math. Ann. 268 (1984), 347-355.

9. M. DE WILDE, Closed graph theorems and webbed spaces (London, Pitman, 1978).

10. S. DIEROLF, On spaces of continuous linear mappings between locally convex spaces (Habilitationsschrift, Munich, 1983).

11. K. FLORET, Some aspects of the theory of locally convex inductive limits, Functional Analysis, Surveys and Recent Results II, K. Bierstedt, B. Fuchsteiner (ed.) (Amsterdam-New YorkOxford, North-Holland, Mathematics Studies 38, 1980). 
12. A. Grothendieck, Sur les espaces (F) et (DF), Summa Brasil Math. 3 (1954), 57-123.

13. A. Grothendeck, Produits tensoriels topologiques et espaces nucléaires (Mem. Amer. Math. Soc. 16, 1955).

14. R. Hollstein, (DCF)-Raüme und lokalkonvexe Tensorproducte, Arch. Math. 29 (1977), $524-531$.

15. R. Hollstern, Inductive limits and $\varepsilon$-tensor products, J. reine. angew Math. 319 (1980), 3862.

16. R. Hollstein, $\otimes$-sequences and inductive limits with local partition of the unity, Manuscripta Math. (to appear).

17. H. JARCHOw, Locally convex spaces (Stuttgart, B. G. Teubner, 1981).

18. G. KöTHE, Topologocal vector spaces I and II (Berlin-Heidelberg-New York, Springer, 1969 and 1979).

19. J. M. Mazón, Some classes of locally convex spaces, Arch. Math. 38, (1982), 131-137.

20. J. Mendoza, Barrelledness on $c_{o}(E)$, Arch. Math. 40 (1983), 156-158.

21. J. Mujica, Spaces of continuous functions with values in an inductive limit, Functional Analysis, Holomorphy and Approximation Theory (Lecture Notes in Pure and Applied Math. 83 1983).

22. J. MuJICA, Domains of Holomorphy in DFC spaces, Functional Analysis, Holomorphy and Approximation Theory, Machado (ed.), (Lecture Notes in Math, 843, Berlin-Heidelberg-New York Springer, 1981), 500-533.

23. W. Roelcke, and S. Dierolf, On the three-space-problem for topological vector spaces, Collect. Math. 32 (1981), 13-35.

24. W. Ruess, Compactness and collective compactness in spaces of compact operators, $J$. Math. Anal. Appl. 84 (1981), 400-417.

25. W. Ruess, Weakly compact operators and (DF)-spaces, Pacific J. Math. 98 (1982), 419441.

26. M. VAldivia, Sur certains hyperplans qui ne sont pas ultrabornologiques dans les espaces ultrabornologiques, C.R. Acad. Sc. Paris 284 A (1977), 935-937.

27. M. VAldivia, A class of locally convex spaces without C-web, Ann. Inst. Fourier 32 (1982), 261-269.

28. M. VAlDiviA, Topics in locally convex spaces (Amsterdam-New York-Oxford: North Holland, Mathematics Studies 67, 1982).

Departamento de Matematicas

E.T.S.I. INDUSTRIALES

Universidad Politecnica

C. DE VERA

VALENCIA

SPAIN 